\title{
A lipocentric view of peptide-induced pores
}

\author{
Gustavo Fuertes • Diana Giménez • \\ Santi Esteban-Martín • Orlando L. Sánchez-Muñoz • \\ Jesús Salgado
}

Received: 8 November 2010/ Accepted: 3 March 2011/Published online: 26 March 2011

(C) The Author(s) 2011. This article is published with open access at Springerlink.com

\begin{abstract}
Although lipid membranes serve as effective sealing barriers for the passage of most polar solutes, nonmediated leakage is not completely improbable. A high activation energy normally keeps unassisted bilayer permeation at a very low frequency, but lipids are able to selforganize as pores even in peptide-free and protein-free membranes. The probability of leakage phenomena increases under conditions such as phase coexistence, external stress or perturbation associated to binding of nonlipidic molecules. Here, we argue that pore formation can be viewed as an intrinsic property of lipid bilayers, with strong similarities in the structure and mechanism between pores formed with participation of peptides, lipidic pores induced by different types of stress, and spontaneous transient bilayer defects driven by thermal fluctuations. Within such a lipocentric framework, amphipathic peptides are best described as pore-inducing rather than pore-forming elements. Active peptides bound to membranes can be understood as a source of internal
\end{abstract}

Membrane-active peptides: 455th WE-Heraeus-Seminar and AMP 2010 Workshop.

G. Fuertes · D. Giménez · S. Esteban-Martín .

O. L. Sánchez-Muñoz · J. Salgado $(\bowtie)$

Instituto de Ciencia Molecular, Universitat de València, C/Catedrático José Beltrán, 2, 46980 Paterna Valencia, Spain e-mail: jesus.salgado@uv.es

J. Salgado

Departamento de Bioquímica y Biología Molecular,

Universitat de València, C/Doctor Moliner, 50,

46100 Burjassot Valencia, Spain

Present Address:

S. Esteban-Martín

Institute for Research in Biomedicine,

Parc Científic, C/Baldiri Reixac 10, 08028 Barcelona, Spain surface tension which facilitates pore formation by diminishing the high activation energy barrier. This first or immediate action of the peptide has some resemblance to catalysis. However, the presence of membrane-active peptides has the additional effect of displacing the equilibrium towards the pore-open state, which is then maintained over long times, and reducing the size of initial individual pores. Thus, pore-inducing peptides, regardless of their sequence and oligomeric organization, can be assigned a double role of increasing the probability of pore formation in membranes to high levels as well as stabilizing these pores after they appear.

Keywords Lipidic pore - Antimicrobial peptide . Pore-forming proteins $\cdot$ Pore energetics $\cdot$ Membrane permeability $\cdot$ Pore structure

\section{Introduction}

In functional studies of biomolecules, proteins and peptides (polypeptides in general) usually take the starring role. This is due to various reasons, such as the wide range of properties encoded by the side-chains of amino-acid residues, which gives polypeptides extraordinary potential for structure and function, and the direct link between the polypeptide sequence and genetic information. In the case of biological membranes, polypeptides occupy also a predominant position in terms of function. Thus, apart from some cases where a particular lipid, usually attached specifically to a protein, exerts a recognized function (Lee 2004), lipids (or the lipid bilayer) are relegated to a secondary role or merely considered as a solvent. Much of this conception is connected to the predominant view after Singer and Nicolson's fluid mosaic model (Singer and 
Nicolson 1972), where the lipidic part of the membrane is depicted as a two-dimensional liquid. An alternative view, however, regards biological membranes as dynamic supramolecular complexes, where lipids are active components even in functions usually assigned to proteins (Fuertes et al. 2010b). The lipids impose specific physical properties on the bilayer, determining its degree of order, fluidity, and thickness, and in some cases being responsible for the formation of domains (Simons and Vaz 2004). The bilayer lipid arrangement is also crucial as a docking surface for membrane-bound molecules, facilitates the proper restructuring and reorganization needed for active membrane proteins, and influences protein-protein interactions (White et al. 2001). Lipids can thus function as a regulatory factor in biomembrane-active complexes, and cells "know" how to "use" them to modulate membrane activity, e.g., varying the lipidic composition depending on conditions (DeLong and Yayanos 1985; Avery et al. 1995). Examples illustrating such a renewed protagonism of lipids include membrane-mediated protein-protein interactions (GarcíaSáez et al. 2009), enzyme and channel activation controlled by mechanical properties of the membrane (Jensen and Mouritsen 2004; Phillips et al. 2009), and the proposed influence of lipid channels for the propagation of nerve pulses (Heimburg and Jackson 2005; Andersen et al. 2009).

For the pore-forming function assigned to amphipathic peptides, lipids put also their peculiar physiochemical characteristics into action. Although such properties are generally broad and common to different types of lipids, they can also be very specific; for example, lipids exhibiting comparable shape may impose similar monolayer curvature and thus have a similar effect on pore formation. However, the special phase, shape, and dynamics characteristics of particular lipids, such as cholesterol, may confer them with specific roles in pore formation and/or activity (Fuertes et al. 2010b). Notice that from the peptide side, pores are weakly specific phenomena, loosely codified at the sequence level, as is shown by the large number and diversity of peptides exhibiting similar pore activities over multiple types of membranes. In fact, structure-function relationships for these peptides appear to follow special rules, based on interfacial activity (Wimley 2010). Such rules, which have yet to be decoded, seem to be best expressed by physicochemical balances of peptide properties (Rathinakumar and Wimley 2008), such as hydrophobicity and amphipathicity, which are also important properties of lipids.

Pores are natural phenomena in lipid membranes, where they may exist even in the absence of proteins or peptides. An interesting manifestation of the intrinsic capacity of bilayer lipids to self-organize to form pores is the increased permeability of membranes specifically at, and near, the phase-transition temperature. As explained recently in an extensive review (Heimburg 2010), these so-called lipid channels exhibit quantized conductance and may be inhibited by drugs, being in fact very similar to (and even indistinguishable from) protein channels. Throughout this review we describe and explain membrane pores from the perspective of lipids. We believe that this lipocentric view is useful and complementary to the usual peptido/proteocentric perspectives and helps understanding critical aspects of pore formation, structure, and stability. We argue that purely lipidic pores and peptide-induced pores are mechanistically related, and similar arguments may indeed be extended to protein-induced pores.

\section{Intrinsic leakage of membranes}

Order, defects, and the passive permeability of bilayers

Lipid membranes act as barriers that separate compartments in aqueous solvents. Their structure corresponds to a lamellar bilayer which self-organizes due to the hydrophobic effect. However, contrary to what is often assumed, lipid membranes are not perfectly sealing or insulating media. They are semipermeable and, even in the absence of proteins, may allow passive transport of small polar and ionic molecules (Deamer and Bramhall 1986; Finkelstein 1987; Jansen and Blume 1995; Paula et al. 1996; Mathai et al. 2009). Such an intrinsic dichotomy may have been crucial at early stages of life (before proteins came onto the scene) (Meierhenrich et al. 2010), because communication with the outside is as important as homeostatic control of the interior of cell compartments. For example, passive diffusion of water across pure lipid membranes, i.e., apart from the contribution of specialized protein channels called aquaporins, is relatively fast and contributes to the osmotic pressure of cells. Small ions can also permeate across membranes, although with much slower diffusion (equilibrating in days). For these cases, the large difference in the electrostatic free energy between the bulk water solution and the membrane hydrophobic core implies that ion transport should occur with the participation of hydrated gateways. However, such structures can also form spontaneously.

\section{Models of passive permeability}

The mechanisms of passive permeation of polar and charged molecules across purely lipidic membranes are still not well understood (Paula et al. 1996; Heimburg 2010). Early experiments and theoretical considerations suggest that water and other small, uncharged, polar molecules (such as glycerol and urea) may follow a solubilitydiffusion mechanism (Paula et al. 1996; Nagle et al. 2008), which is described as a continuum model that considers 
partition of the solutes between the bulk water and a membrane hydrophobic slab (Fig. 1a). However, water and polar molecules most likely permeate through some sort of pores (Jansen and Blume 1995), which in the most general sense can be considered as bilayer edge defects. The simplest of these and the easiest to be formed, since it involves minimal distortion of the lipids, corresponds to so-called water files or wires (Finkelstein 1987), which are single rows of water molecules flowing across the bilayer (Fig. 1b). These structures are often observed in molecular simulations and are related to incipient (narrow) states of hydrophobic pores (walled by the acyl chains of lipids) which are the pre-pore states proposed by Glaser et al. (1988). On the other hand, ions move across membranes without completely losing their hydration shell. In some molecular dynamics (MD) simulations they have been seen to pass intercalated within the water files (Gurtovenko and Vattulainen 2007; Böckmann et al. 2008) (see below). In other MD studies (Wilson and Pohorille 1996) ions translocate by means of local thinning defects filled with water which alternate between the two monolayers (Fig. 1c). Both types of defects can be considered precursors of larger hydrophilic pores (Gurtovenko and Anwar 2007; Böckmann et al. 2008), through which ions would pass at low free energy cost and where the walls are made of polar head-groups of lipids (Fig. 1d). This latter structure is often named a toroidal pore and involves transient reorientations of some lipids at the membrane border, as first hypothesized by Glaser et al. (1988), who also proposed that hydrophilic pores derive from short-lived intermediate hydrophobic pores after they reach a critical radius (a mechanism described for electroporation but probably having general validity; see below).

The role of membrane dynamics

Experiments and simulations are making it increasingly clear that intrinsic membrane permeability is linked to the complex structure and dynamics of lipid bilayers, as illustrated in Fig. 2a, which shows a snapshot from a selfassembly MD simulation (Esteban-Martín and Salgado 2007). The regions of the membrane occupied by polar groups in the two monolayers, known as the interfaces, are highly hydrated, and together are roughly as thick as the hydrophobic center slab (White and Wimley 1999). However, the positions of polar (including water) and hydrophobic groups are spatially and temporally highly variable, being best described by broad distributions (Fig. 2b). This was first shown by Wiener and White (1991, 1992) from the interpretation of X-ray diffraction and neutron diffraction data and can be fairly well reproduced by MD simulations. As a consequence, at any particular moment, some lipids can be found displaced with respect to their
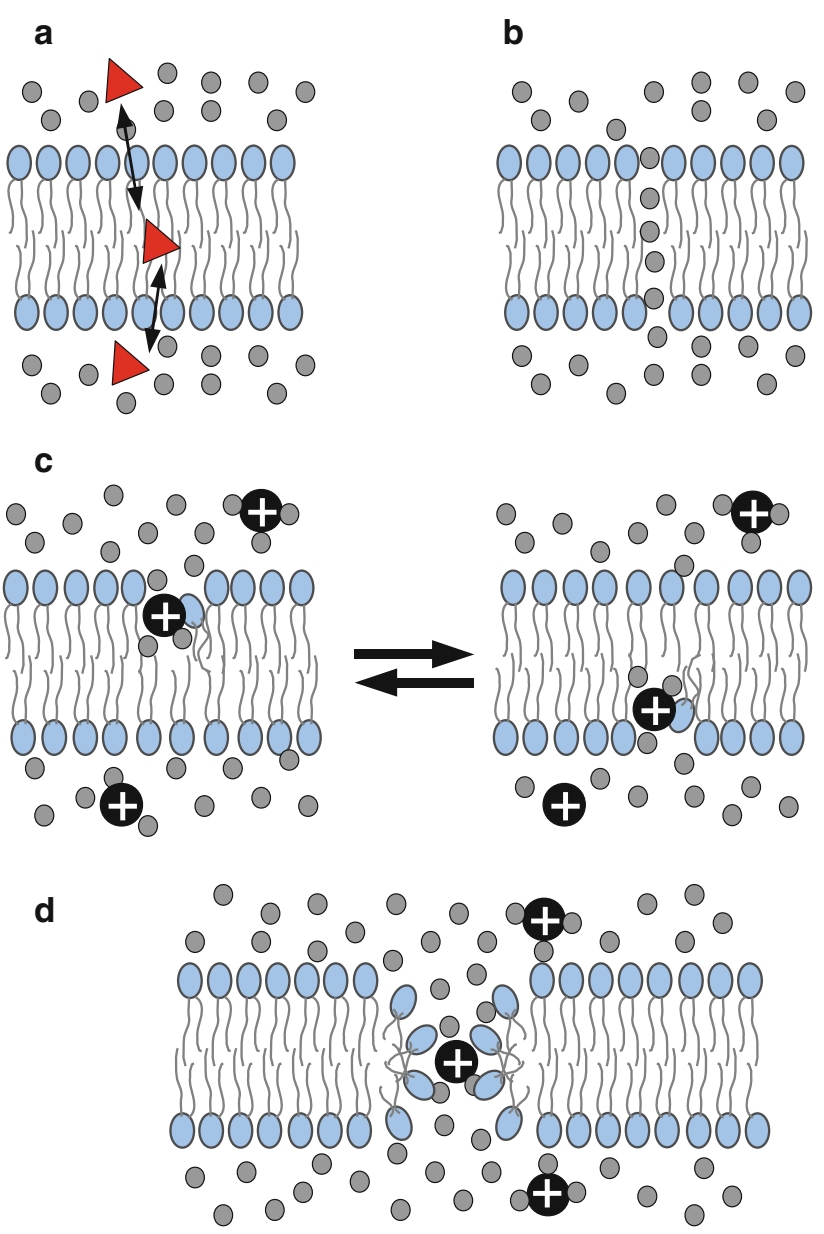

Fig. 1 Schematic representation of simple models explaining passive diffusion of small polar molecules, such as water, and ions across membranes. a The solubility-diffusion model involves simple partitioning equilibria of the transported molecule without lipid rearrangement. This mechanism explains diffusion of nonpolar and some polar organic molecules (Paula et al. 1996). b Water is believed to cross membranes through special pores in the form of single-row alignments called water files or wires (Finkelstein 1987; Böckmann et al. 2008), which involve only mild reorganization of lipids and correspond to the hydrophobic pores hypothesized by Glaser et al. (1988). c Ions might move within the water wires, but most likely (and readily) permeate through larger hydrophilic pores (d), with walls lined by lipid head-groups, formed by reorientation of some lipids at the pore rim (Glaser et al. 1988)

average structure, causing local thinning of the hydrophobic core, with water also penetrating deep (Fig. 2a). Such dynamic/transient rearrangements are driven by thermal fluctuations (Glaser et al. 1988; Jansen and Blume 1995; Nagle and Scott 1978). They originate local defects in the smectic liquid-crystal order of lipids which approximate to the transition state for passive diffusion of polar molecules across the membrane (Wilson and Pohorille 1996). At arbitrary times and regions, defects may nucleate and give rise to transient water-filled pores (as in Fig. 1d) which may act as freeways for the passage of ions. Under normal 
conditions, such distortions are unfavorable, stochastic, short-lived, low-probability events. However, given the very large number lipids in a membrane (even for small vesicles), and their fast dynamics and large fluctuations in the liquid-crystal state, passive permeability occurs and is measurable in unperturbed vesicles (Deamer and Bramhall 1986; Paula et al. 1996).

Permeation is enhanced at phase transition

Because the intrinsic membrane leakage proceeds via bilayer defects, it is expected to be more likely at conditions of higher probability of defects, such as at phase coexistence (Nagle and Scott 1978). This is indeed the case, since passive permeability of lipid bilayers to water (Jansen and Blume 1995), cations (Papahadjopoulos et al. 1973), and large polar solutes (Blicher et al. 2009) exhibits a maximum near the gel to liquid-crystalline phase transition, even in pure dipalmitoylphosphatidylcholine (DPPC) membranes. These phenomena were discovered early, both in macroscopic permeability experiments performed with ensembles of vesicles (Papahadjopoulos et al. 1973; Jansen and Blume 1995) and in single-channel recordings measured by electrophysiology (Yafuso et al. 1974; Antonov et al. 1980; Yoshikawa et al. 1988; Kaufmann et al. 1989). The purely lipidic ion channels are found to be very similar to protein channels. They exhibit quantized currents corresponding to large pores with a

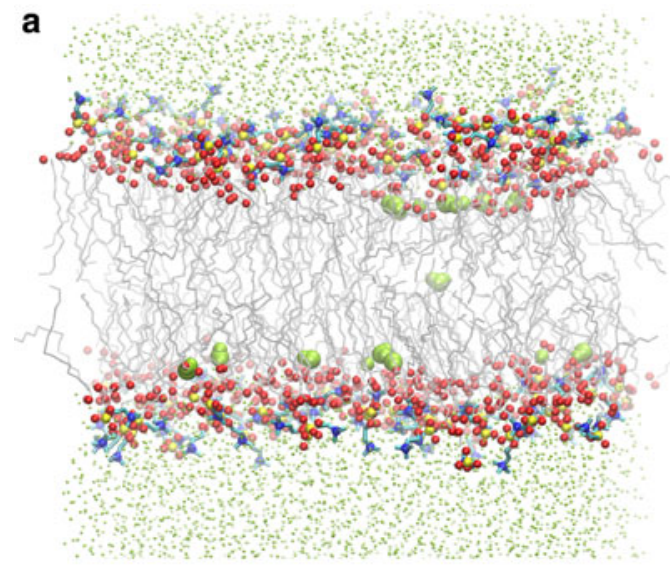

Fig. 2 Example of a simulated self-assembled and equilibrated DPPC lipid bilayer. The data correspond to a control MD simulation at $323 \mathrm{~K}$ reported by Esteban-Martín and Salgado (2007) a. Acyl chains of lipids are represented by gray lines, head-group atoms are balls colored red (oxygen) blue (nitrogen), and yellow (phosphorus); water is colored green, as small balls for molecules in typical hydrated regions, and as a CPK representation for molecules penetrating deeper (within a $20-\AA$ center slab). Although lipid atoms tend to occupy confined positions as dictated by the hydrophobic effect and corresponding to the positional and orientational order of a smectic liquid-crystal phase, the bilayer is characterized by large spatial disorder. Notice, for example, the deeper penetration of some narrow distribution of sizes (Antonov et al. 1980, 2005; Boheim et al. 1980) and moderate ion selectivity (Antonov et al. 2005). The presence of lysolipids at the gel-to-liquid phase transition dramatically enhances membrane leakage, and this is proposed to occur through lysolipid-stabilized pores in the boundary of the partially melted solid phase (Mills and Needham 2005). On the contrary, permeability is inhibited in DPPC membranes in the presence of cholesterol, in parallel with the clearance of the chain melting transition (Papahadjopoulos et al. 1973). Although these lipid ion channels (Heimburg 2010) were recognized as a problem for the interpretation of experiments with proteins, and were suggested as physiologically relevant for conduction of current in biological membranes (Yafuso et al. 1974; Antonov et al. 1980), membrane permeability continues to be largely assigned to proteins and peptides.

Through a series of detailed investigations (Seeger et al. 2005; Wodzinska et al. 2009; Blicher et al. 2009; Gallaher et al. 2010; Heimburg 2010), the group of Heimburg has recently revitalized the study of lipid ion channels, for which they have proposed new and exciting physiological relevance for nerve pulse propagation and the action of anesthetics (Heimburg and Jackson 2005; Heimburg and Jackson 2007; Andersen et al. 2009). Using a new theory of membrane fluctuations, Heimburg describes the likelihood of lipid channels with respect to temperature, pressure, tension, and other thermodynamic variables, as well as voltage, $\mathrm{pH}$, ions, drugs (such as anesthetics and

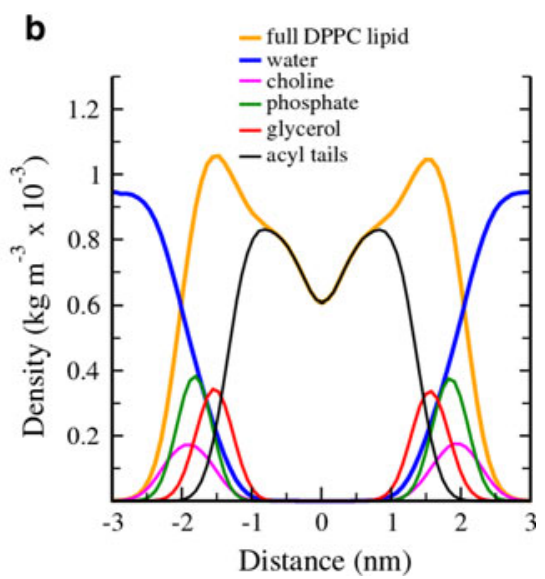

polar lipid head-groups and of a few water molecules. Furthermore, such positions are highly variable with time and are best described by broad distributions, represented in $\mathbf{b}$. Although positions of water and polar groups reaching deep within the hydrocarbon region are of very low probability, they occur as transient states which can be the source of local thinning fluctuations and eventually facilitate pore formation. The distributions in $\mathbf{b}$ were calculated by averaging the latest $2 \mathrm{~ns}$ in the trajectory of the equilibrated self-assembled bilayer (20 ns after complete closure of the pore that forms during aggregation), while the picture in a corresponds to a selected frame within the same time range 
neurotransmitters), and proteins (Heimburg 2010). All of these can in fact be considered perturbation factors that influence the melting transition regime. In addition, he demonstrates via the fluctuation-dissipation theorem that the dwell time of fluctuations and the lifetime of pores are closely coupled (Heimburg 2010). The link between membrane permeability and phase transition is related to the coexistence of domains, a condition where area fluctuations, heat capacity, and compressibility are large. Nagle and Scott (1978) reasoned that the size of defects (pores), $\Delta A$, is proportional to the compressibility $\kappa_{\mathrm{T}}^{\mathrm{A}}$ as $(\Delta A)^{2} \propto \kappa_{\mathrm{T}}^{\mathrm{A}}$, and they related the membrane permeability at phase transition $(P)$ with that of a pure phase $\left(P_{0}\right)$ and the (fluctuating) size of defects, according to $P=P_{0}+C(\Delta A)^{2}=P_{0}+C^{\prime} \kappa_{\mathrm{T}}^{\mathrm{A}}$ (with $C$ and $C^{\prime}$ being proportionality constants). In turn, Heimburg derives that, for transitions of lipid membranes, the changes of compressibility are proportional to the excess heat capacity, from which it follows that $P=P_{0}+\alpha \Delta C_{\mathrm{p}}$, where $\alpha$ is a constant (Heimburg 2010). Such predicted proportionality between permeability and heat capacity is nicely confirmed by experiments (Blicher et al. 2009). The increased membrane permeability must be attributed to the overall membrane fluctuations. Monte Carlo simulations of binary lipid mixtures at the melting regime for dimiristoylphosphatidylcholine:distearoylphosphatydylcholine (DMPC:DSPC) $1: 1$ at $36.9^{\circ} \mathrm{C}$ show lateral domain separation with larger local fluctuations in fluid than in gel domains which are maximal in narrow areas at the domain interfaces (Seeger et al. 2005). Thus, it can be expected that pores form predominantly at interfaces, with possibly some contribution of fluid phases to permeability.

Passive lipid flip-flop is coupled to intrinsic membrane permeability

Another complex phenomenon related to membrane dynamics and membrane permeability is transbilayer diffusion of lipids, usually known as flip-flop. Although in cell membranes this process is catalyzed by flipases (Daleke 2007), it can also occur spontaneously even in protein-free membranes (on time scales of hours to days, depending on conditions) (Kornberg and McConnell 1971; Wimley and Thompson 1990; De Kruijff and Van Zoelen 1978; Nakano et al. 2009). Unassisted flip-flop has been proposed to be activated by lipid-packing defects, driven by thermal fluctuations (Wimley and Thompson 1991; De Kruijff and Van Zoelen 1978), and the experimentally determined activation energy for lipid flip-flop (Kornberg and McConnell 1971) is similar to the value measured for spontaneous chloride permeation (Toyoshima and Thompson 1975). In line with these data, lipid flip-flop in MD simulations is mediated by transient water pores, which facilitate translocation of the polar head-groups across the membrane in a solvated state (Tieleman and
Marrink 2006; Gurtovenko and Vattulainen 2007a), altogether suggesting that flip-flop coupled pores may be responsible for passive permeation of ions (Toyoshima and Thompson 1975; Tieleman and Marrink 2006; Gurtovenko and Vattulainen 2007a). However, flip-flop pores would represent a negligible contribution to the transbilayer diffusion of water (Tieleman and Marrink 2006), in agreement with the existence of multiple alternative pathways for water permeability.

On the other hand, unassisted lipid flip-flop is enhanced at phase transition (De Kruijff and Van Zoelen 1978), in a way similar to described above for passive membrane permeability. In fact, both phenomena appear to be affected by the same membrane composition and perturbation factors; for example, ceramide facilitates flip-flop as well as the formation of large and stable lipidic channels (RuizArgüello et al. 1996; Siskind and Colombini 2000; Contreras et al. 2005). Interestingly, MD simulations also show that asymmetric transbilayer distributions of ions (Gurtovenko and Vattulainen 2005, 2007a, Gurtovenko and Anwar 2007) and/or lipids (Gurtovenko and Vattulainen $2007 b$ ) originate mild membrane potentials which are sufficient to drive water-pore defects, allowing lipid flipflop and ion transport. These latter studies are important because they correspond to internal stress conditions which might be easily achieved at physiological states of membranes.

The presence of proteins and peptides bound across the membrane, even when they do not form pores per se, is able to increase lipid flip-flop. Such is the case of glycophorin (de Kruijff et al. 1978), some $\alpha$-helical membrane proteins from the membrane of bacteria (Kol et al. 2003), and synthetic model transmembrane peptides (Kol et al. 2001; Sapay et al. 2010). Protein-facilitated flip-flop has been proposed to occur through a reduction of the free energy of phospholipid desorption as well as the barrier for defect (water-pore) formation (Sapay et al. 2010). Transmembrane proteins may also alter the phase behavior of the lipid membrane (Freire et al. 1983; Morrow et al. 1986; Heimburg 2007). These facts are used to propose a proteinmediated membrane perturbation mechanism via hydrophobic mismatch that would create small belts of highly fluctuating, defect-prone lipids at the interface with proteins (Heimburg 2010). Such a mechanism is supported by Monte Carlo simulations of gramicidin A under negative mismatch in model DPPC membranes in the gel state (Ivanova et al. 2003).

\section{Lipid pores induced by tension}

The spontaneous lipid pores described in the previous section are handicapped by a large energy barrier. This 
corresponds to the formation of a transition state that consists of a type of edge defect generally termed a water pore. Our discussion above also involves that such an activation energy for pore formation can be lowered at conditions of increased fluctuations, such as near the membrane melting regime or by perturbations due to membrane inclusions (such as peptides and proteins). Similarly, pores can be induced by applying different types of external tension, on the order of $\sim 1-25 \mathrm{mN} / \mathrm{m}$ (Bloom et al. 1991). Mechanical (Zhelev and Needham 1993; Sandre et al. 1999; Evans et al. 2003), osmotic (Taupin et al. 1975) or electrical tension (Benz et al. 1979; Tsong 1991 ) is most often employed. One can easily reason that what such an external tension does is, again, to increase area and compressibility fluctuations, thereby increasing the heat capacity [according to Heimburg's (2010) framework theory] and reducing the pore activation energy. There are multiple examples relating spontaneous pores (such as lipid channels and flip-flop pores) with pores induced by tension; for example, pure lipid channels are mechanosensitive, since they appear after suction on a patch pipette (Kaufmann et al. 1989). They are also voltage - sensitive, and in fact the reversible electrical breakdowns of artificial membrane conductance produced at high voltages (Benz et al. 1979; Glaser et al. 1988) are phenomena closely related to electroporation of lipid vesicles or cells (Ryttsén et al. 2000). On the other hand, both experiments (Schwarz et al. 1999) and simulations (Kandasamy and Larson 2006) show that lipid flip-flop can be enhanced by applied external electric fields. Tensioninduced pores have important applications for biotechnology and medicine (Neumann et al. 1982; Tsong 1991; Bodles-Brakhop et al. 2009), which has motivated intense investigation of these phenomena during the last decade. Sophisticated experimental setups have allowed detailed studies of pore kinetics and thermodynamics (Zhelev and Needham 1993; Moroz and Nelson 1997; Sandre et al. 1999; Ahmad and Evans 2002), while ever-improving MD simulations have provided descriptions at a molecular level (Marrink et al. 2009). Notwithstanding the importance of these recent achievements, the basis for understanding lipid pore formation and evolution were set up at least one decade earlier (Litster 1975; Taupin et al. 1975; Abidor et al. 1979; Benz et al. 1979; Glaser et al. 1988; Melikov et al. 2001). Let us now summarize these seminal ideas

The pore inversion mechanism

A model explaining pore formation and evolution under electrical stress was proposed by Glaser et al. (1988). Reversible electrical breakdowns recorded in planar membranes were interpreted by assuming an initial hydrophobic pre-pore (described above and illustrated in
Fig. 1b) which relaxes into a hydrophilic (inverted) pore of larger radius, characterized by lipid reorganization and participation of some lipids in the pore wall (Fig. 1d). The hydrophobic pre-pore is an intermediate state with energy:

$E_{0}(R)=2 \pi h R \sigma_{0}(R)$

that depends on the membrane thickness $(h)$, the pre-pore radius $(R)$, and an interface-membrane tension, which in turn is a function of the pre-pore size, $\sigma_{0}(R)\left[\sigma_{0}(0)=0\right.$, $\left.\sigma_{0}(\infty)=0.05 \mathrm{~N} / \mathrm{m}\right]$. The latter term is a hydrophobic effect correction on the value of the interface tension, which scales the pre-pore energy to near zero for very small pores. Thus, the hydrophobic pores start at zero energy (for $R=0$ ), remain at low values in the small poresize regime (Fig. 3, red line), and can be simply driven by thermal fluctuations of lipids. As the radius of the pre-pore grows, its energy will eventually increase quickly, up to a critical pore radius $R^{*}$ for which it equals the energy corresponding to a hydrophilic pore of that size: $E_{0}\left(R^{*}\right)=E_{i}\left(R^{*}\right)$. At this stage, the rearrangement of some lipids to conform the hydrophilic pore wall becomes energetically favorable (pore inversion) (Glaser et al. 1988). The energy of the hydrophilic pore can be described by the potential of a hole in a two-dimensional continuum, often referred to as Litster's model (Deryagin and Gutop 1962; Litster 1975; Taupin et al. 1975; Glaser et al. 1988; Evans et al. 2003; den Otter 2009):

$E_{i}(R)=2 \pi \gamma R-\pi \sigma_{i} R^{2}$.

This includes a surface tension $\left(\sigma_{i}\right)$ term (energy per unit pore area) and a line tension $(\gamma)$ or edge energy term (energy per unit length of the pore perimeter, assumed to be circular). The line tension term corresponds to the energy of cohesion, opposing the pore. The surface tension term represents the potential for the mechanical work of expansion of the pore, which originates from a source of stress. Glaser et al. argue that such a continuum description of the hydrophilic pore energy is not valid for small pores $(R \ll h)$, where the free energy should grow steeply because of lipid packing constraints and repulsive hydration interactions (Fig. 3, dashed blue line). Nevertheless, this framework is useful to draw a qualitative energy landscape, which predicts a minimum of $E_{i}$ for $R_{m}$. Beyond that size, but still within an intermediate size regime, the pore tends to shrink back to the minimum size, since the line tension term dominates (Fig. 3, blue solid line). However, for $R>R_{\mathrm{d}}=\gamma / \sigma_{i}$ (the large size regime), the energy barrier $E_{d}=\pi \gamma^{2} / \sigma_{i}$ is overcome and pore expansion becomes indefinitely favorable (i.e., the membrane breaks irreversibly) (Litster 1975; Taupin et al. 1975; Abidor et al. 1979).

Various extensions of Litster's model have been used to describe the stability and evolution of hydrophilic pores in 


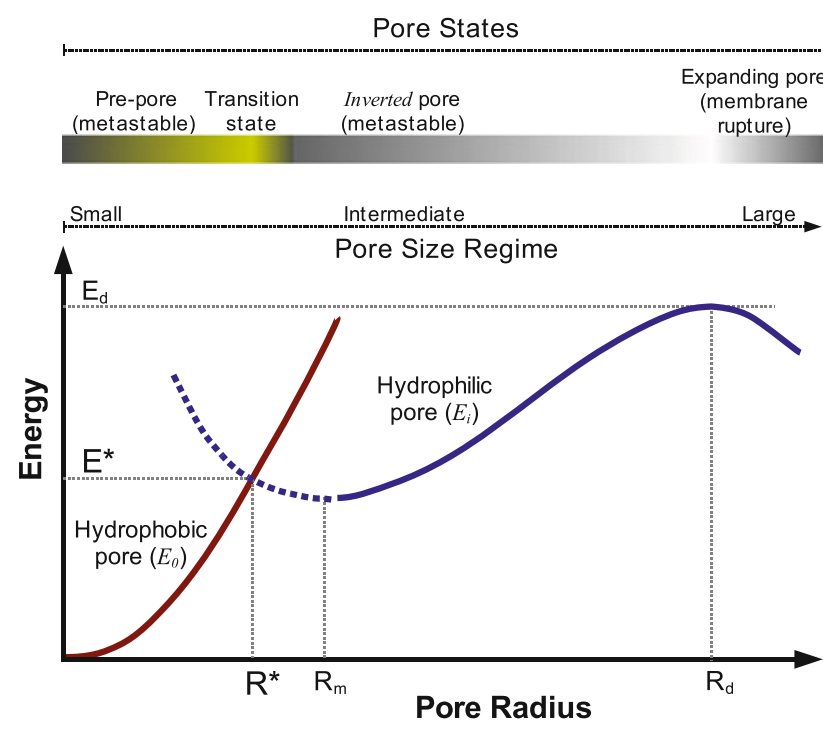

Fig. 3 Free energy of hydrophobic (pre-pore) and hydrophilic pores as a function of pore radius. The graph is a qualitative representation based on the continuum theory described by Glaser et al. (1988) for reversible electrical breakdown. Initial pores (pre-pores) are postulated to be of hydrophobic type (Fig. 1b). At a critical pore radius $R^{*}$, the energy of these pores equals that of a hydrophilic pore (Fig. 1d), formed by reorientation of some lipids to line the pore wall with their head-groups. Then, the energy at that state $\left(E^{*}\right)$ corresponds to a hypothetical nucleation barrier for lipid rearrangement to form a hydrophilic pore. This latter starts with an undefined energy (dashed line), but as the radius increases the energy of the pore can be assumed to follow Litster's theory (Litster 1975; Taupin et al. 1975). At larger pore size, a second critical value is predicted $\left(R_{d}\right)$, beyond which the membrane would irreversibly break

membranes [see, for example, Shillcock and Boal (1996), Evans et al. (2003), Tolpekina et al. (2004b), Farago and Santangelo (2005), and Wohlert et al. (2006)], but for the sake of clarity we will stick to the simpler formulation as in Glaser's work. Despite the limitations of this model, it is enough to infer that pre-pore and pore are metastable states. The value of $E_{0}$ (or $E_{i}$ ) at the critical radius, or simply $E^{*}$ (Fig. 3) represents the activation energy for hydrophilic pore formation, which should be taken as mainly the energy barrier for lipid reorientation at the membrane edge in the pore wall. Glaser et al. estimate a value of $R^{*}=0.5 \mathrm{~nm}$ from electrical recordings in planar lipid bilayers induced by voltage pulses (Glaser et al. 1988). The lifetime of the pre-pore should be on the order of the characteristic time for lipid fluctuations. However, the hydrophilic pore can be expected to exhibit larger metastability, since it is trapped between two energy barriers: $E^{*}$ and $E_{d}$. In a more recent study (Melikov et al. 2001) of the evolution of single voltage-induced pores, nonconductive metastable pre-pores were found between spikes of transient pores. These are supposed to be different from the short-lived hydrophobic pre-pores and hypothesized to consist of small clusters of polar heads of lipids and/or water molecules trapped in the hydrophobic interior (Melikov et al. 2001). Chernomordik and coworkers further hypothesized that small metastable pores and pre-pores found under high electric field are a common characteristic of other types of lipidic pores, such as those formed under strong tension (Zhelev and Needham 1993) or in the presence of nonbilayer lipids (Chernomordik et al. 1985), and also suggested that they are relevant for explaining permeability induced by high local concentrations of membrane-active peptides (see below).

As a general formulation, the pore-inversion mechanism should apply to pores formed under very diverse (perhaps all) sources of tension. These include externally applied tension (mechanical, electrical, osmotic) as well as internal tension, such as that originating from asymmetric distributions of lipids or ions (Gurtovenko and Vattulainen 2005, 2007b) or binding of small surface-active molecules (Gurtovenko and Anwar 2007; Moldovan et al. 2007) or peptides/proteins (Huang et al. 2004; Sapay et al. 2010) (see below). This thus helps understanding of and generalization to a single theoretical framework for all types of tension-induced or defect-mediated (Gurtovenko et al. 2010) pores in membranes, which again stresses the importance of intrinsic lipid bilayer properties in these phenomena.

Stress-induced pores studied by MD simulations

The molecular architecture of lipidic pores and pre-pores is difficult to characterize from experiment. However, detailed structural models are provided by a number of recent computational studies which were able to simulate pore formation and evolution on limited time and space scales with atomistic and coarse-grained detail (Marrink et al. 2009; Gurtovenko et al. 2010). Hydrophilic lipid pores were first observed at atomic resolution as an intermediate state during simulation of bilayer self-assembly (Marrink et al. 2001). As postulated in earlier models (Glaser et al. 1988), these pores had an approximately toroidal shape, with the membrane edge stabilized by headgroups of reoriented lipids, and a diameter of about $1 \mathrm{~nm}$. Very similar pores were described from subsequent MD studies using simulated mechanical or electrical stress conditions (Tieleman et al. 2003; Leontiadou et al. 2004; Tarek 2005; Gurtovenko and Vattulainen 2005). Particularly relevant is the observation in some cases of water files (Gurtovenko and Vattulainen 2005, Gurtovenko and Vattulainen 2007), identified as hydrophobic pre-pore intermediates (Böckmann et al. 2008) (Fig. 4). These are precursor, short-lived hydrophobic pores, formed after 1-1.4 ns of simulation, often by convergence of two water fingers from opposite monolayers (Tieleman et al. 2003; Leontiadou et al. 2004). They are favored by local defects 
in the head-group regions, and their likelihood increases with increasing voltage (Böckmann et al. 2008). These dynamic structures grow to form water pores which eventually relax into a hydrophilic pore by reordering of some lipids to orient their head-groups at the pore wall, showing nice resemblance to the two stages envisioned by Glaser et al. (1988). The reorientation of lipid head-groups appears to be the rate-limiting step. On the other hand, the pores remain stable for relatively long times (more than $200 \mathrm{~ns}$ ) after voltage discharge (Gurtovenko and Anwar 2007), similar to pores formed during membrane self-assembly.

It is noteworthy that lipidic pores simulated under electrical and mechanical tension, pores appearing during simulated self-assembly of bilayers, and pores appearing in simulations of membrane-bound surface-active molecules all exhibit very similar features to pores induced in the presence of pore-forming peptides (Leontiadou et al. 2006; Sengupta et al. 2008; Jean-François et al. 2008).
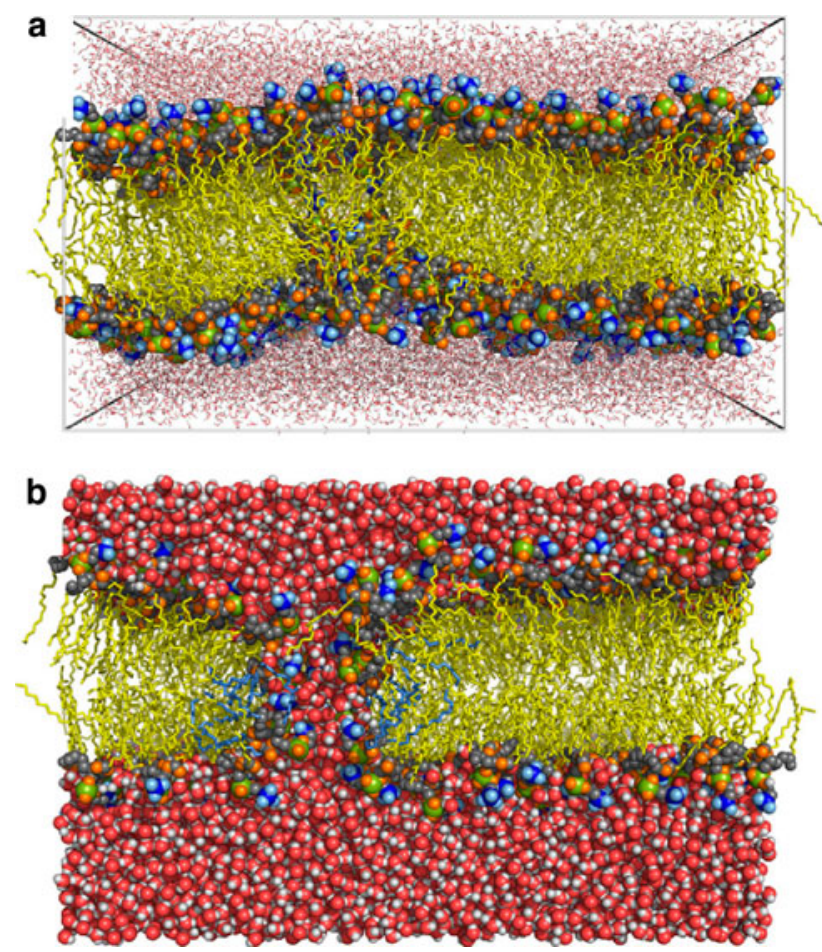

Fig. 4 Pre-pore and pore in trajectories of a simulated bilayer under electrical tension. a Snapshot after $0.9 \mathrm{~ns}$ of MD simulation of a 1-palmitoyl-2-oleoylphosphatidylcholine (POPC) membrane (512 lipids) at electric field of $0.5 \mathrm{~V} / \mathrm{nm}$. Water is drawn as red sticks, lipid acyl chains are colored yellow, choline groups are in blue, phosphorus atoms are in green, lipid oxygens are in orange, and headgroup carbons are in gray. Water-file defects are observed near the middle of the membrane. After $50 \mathrm{~ns}$ of simulation with a decreased field $(V=0.04 \mathrm{~V} / \mathrm{nm})$ the water wire has grown and transformed into a hydrophilic pore, shown in $\mathbf{b}$ (with the same color coding, and water in space-filling representation). The pore has an approximate radius of $\sim 0.47 \mathrm{~nm}$ and is lined by $\sim 8$ lipids (highlighted light blue). Reprinted with permission from Biophysical Journal, Vol. 95 (Böckmann et al. 2008) (C) 2008 Biophysical Society

\section{Peptides come into play: inducers and stabilizers of pores}

We finally come to the usually main actors in the biological pore function, the so-called pore-forming peptides (Zasloff 2002). Throughout the previous sections, peptides and proteins have been mentioned barely and as yet another possible type of perturbation that may increase the likelihood of pores. Do they really play such a secondary role? We will see now that they in fact share protagonism with lipids, although the role that they play may be substantially different from that considered to date. As we have mentioned, proteins can exert perturbing effects by affecting the phase properties of neighboring lipids, like in the case of lipid channels linked to transitions and phase coexistence (Weinstein et al. 1981; Heimburg 2010), or by reducing the activation energy for the transition state of spontaneous pores, as in the case of peptide/protein-enhanced lipid flipflop (Sapay et al. 2010). In their revealing paper, Glaser et al. stated that "by becoming involved in the pore edge, proteins in membranes are expected to slow down pore resealing," adding that "pore formation may be facilitated at lipid-protein junctions" (Glaser et al. 1988). This implies that plausible general effects of membrane proteins and peptides are both easing pore formation and stabilizing pores once they are formed. Can we maintain the same arguments for the well-known, highly pore-active cationic/ amphipathic peptides? It should not escape us that so-called pore-forming peptides and proteins can be seen as specifically designed to make use of their background membraneperturbing tendency as the basis for their specific role.

A link between metastable pores induced by electroporation in protein-free bilayers and lipidic pores induced by peptides and proteins has been suggested by Chernomordik (Melikov et al. 2001) and by Heimburg (2010). On the other hand, Huang et al. (2004) go one step further and adopt Litster's theory to explain the stability of lipid pores induced by peptides. According to this interpretation, the peptides may act simultaneously as a source of internal surface tension and as a relief of line tension, the former favoring pore formation and the latter opposing pore closure. Membrane-active peptides then work essentially as surface-active molecules, able to partition preferentially to the membrane interface (Wimley 2010). The interfacial peptide accumulation produces expansion (Longo et al. 1998; Lee et al. 2008) and thinning (Ludtke et al. 1995) of the bilayer, up to a point where pore formation is no longer kinetically hindered. In Huang's words, "area expansion creates a stress equivalent to a membrane tension," which he then terms internal membrane tension, $\sigma$ (Huang et al. 2004). Such internal tension is expressed as a function of $P / L$ (the peptide-to-lipid molar ratio): $\sigma=K_{a}\left(A_{P} / A_{L}\right)(P / L)$, with $K_{a}$ being the stretching modulus, $A_{P}$ being the area 
increase per peptide molecule, and $A_{L}$ the area per lipid molecule. $\sigma$ is estimated to be in the range corresponding to tension-induced pores: $5-15 \mathrm{mN} / \mathrm{m}$. In this sense, the role of active peptides does not differ much from that of other surface-active (amphiphilic) molecules able to bind readily to membranes and increase their permeability. A simple illustrative case is that of dimethyl sulfoxide (DMSO), studied by MD simulations (Gurtovenko and Anwar 2007; Moldovan et al. 2007), which induces pores through a mechanism very similar to the one described above for membranes under tension. The extensive interaction of DMSO molecules at the membrane interface causes area expansion and a concomitant reduction of thickness, which according to Huang's model is a source of internal tension. This is accompanied by structural defects and water penetration driven by thermal fluctuations, growing to a point where lipid reorientation gives rise to hydrophilic pores (Gurtovenko and Anwar 2007; Moldovan et al. 2007).

On the other hand, using an extension of Lister's theory (Litster 1975; Tolpekina et al. 2004a) it is shown that a reduction of the line tension of the membrane in the presence of DMSO, below a threshold value, facilitates pore nucleation (Moldovan et al. 2007). In experiments with giant unilamellar vesicles (GUV) it is also found that detergents reduce the line tension of pores (Puech et al. 2003). A similar pore-stabilizing effect via line tension attenuation is also hypothesized by Huang as a second characteristic role of the active peptides. From this, he estimates the radius of a stable pore to be in the range $1-2 \mathrm{~nm}$ for the case of magainin in DMPC:DMPG (3:1) membranes (Huang et al. 2004), coinciding with measurements by neutron diffraction (Ludtke et al. 1996). Evidence of line tension reduction by active peptides has been obtained in the presence of the Bax- $\alpha 5$ fragment. This peptide originates shape changes and coalescence of liquidordered domains in phase-separated bilayers, as observed by fluorescence microscopy of GUVs and in situ atomic force microscopy (AFM). The line tension changes were further analyzed in AFM film-rupture experiments (GarcíaSáez et al. 2007) and agree with results of another AFM study of membrane remodeling for a variety of poreforming and cell-penetrating peptides (Shaw et al. 2008). Thus, pores stand as a response of the bilayer to peptideinduced internal tension, and the peptide further helps by maintaining the pore stable and with a defined size (Huang et al. 2004; Lee et al. 2008; Ludtke et al. 1996).

\section{Structure of pores induced by peptides}

There is now a growing consensus around the idea that the pores stabilized by most cationic membrane-active peptides are of toroidal type (Zasloff 2002; Almeida and Pokorny 2009; Fuertes et al. 2010b). These pores correspond to Glaser's hydrophilic pore (Glaser et al. 1988), defined by Matsuzaki as a supramolecular peptidelipid dynamic complex (Matsuzaki et al. 1996) and by Huang as the worm-hole model (Ludtke et al. 1996). Direct evidence and a low-resolution structure for such a lipidbased arrangement, in this case for pores induced by the $\alpha 5$ active fragment of the protein Bax (García-Sáez et al. 2005), have been provided by grazing-angle X-ray diffraction using supported bilayers with brominated lipids (Qian et al. 2008b) (Fig. 5). The lipid electron density clearly shows a circular pore with toroidal geometry, with the monolayers fused at the pore wall. Two facts should be realized from this structure: (1) It corresponds to an equilibrium state, with equal distribution of peptides across the membrane (in the two monolayers), since the membranes were prepared by reconstituting lipids and the peptide from homogeneous mixtures; and (2) It provides information only about the lipidic membrane edge of the pore, since the analyzed diffraction data correspond only to the electrodense brominated lipids. Thus, the model unfortunately gives no clue about the arrangement of peptides, which are assumed to bind near the pore wall. The relative orientation of the peptide in the membrane is taken as that inferred from changes of the circular dichroism spectrum in oriented bilayers as a function of the $P / L$ ratio beyond a threshold value $(P / L)^{*}$, which occur in parallel to membrane thinning (Ludtke et al. 1996). Interestingly, recent calculations, using an implicit membrane model, predict preferential binding of various cationic antimicrobial peptides, with an oblique orientation, at the edge of toroidal pores, which seems to be related to their imperfect amphipathicity (Mihajlovic and Lazaridis 2010).

On the other hand, simulated structures of peptideinduced pores have been obtained by MD at atomic resolution (Leontiadou et al. 2006; Sengupta et al. 2008; JeanFrançois et al. 2008), which although agreeing essentially with the toroidal model, are rather disordered in shape and for the arrangement of lipids and peptides. However, the MD studies simulate principally an asymmetric peptide attack to the membrane, and the reported structures correspond to snapshots or small time windows of individual pores. Multiscale simulations, combining coarse-grained and atomistic resolution, were also performed and reached up to tens of microseconds (Thogersen et al. 2008; Rzepiela et al. 2009), but this time scale is still very far from the equilibration time inferred from experiments with single vesicles (Fuertes et al. 2010a), and the simulated pores likely correspond to intermediate states. Therefore, the pores from MD simulations are not directly comparable to the equilibrium experimental structures (Qian et al. 2008b), but rather with kinetic pores formed at short time after peptide biding to vesicles (Yandek et al. 2007; Gregory et al. 2008; Fuertes et al. 2010a). Nevertheless, the MD trajectories have great value 

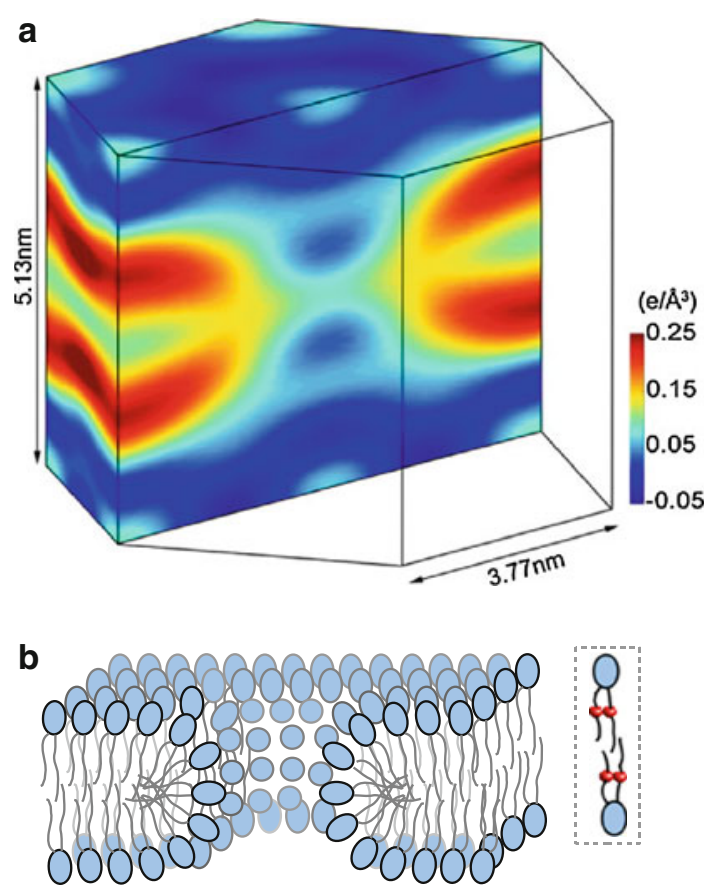

Fig. 5 Bilayer structure at the edge of a peptide-induced lipid pore. The lipidic outline of the pore is viewed from the electron density of brominated lipids, resolved by grazing-angle X-ray diffraction (Qian et al. 2008b) a. The structure corresponds to the pore induced by the $\alpha 5$ active fragment of the protein Bax. It shows continuous electron density between the two monolayers at the pore edge, as expected for the case of toroidal pores with a wall lined (at least partially) by lipids, drawn schematically in $\mathbf{b}$, where the bromine labels in the acyl chains of lipids are represented as small balls in lipids within a dashed-line box. Because these experiments do not provide data about the peptide part, we have purposely omitted it from the cartoon. A different pore-edge structure, with no participation of lipid headgroups in the wall, was found for the pore formed by alamethicin (a nonionic amphipathic peptide), which is in agreement with a barrelstave model (Qian et al. 2008a). However, such a type of pore does not appear adequate for the majority of pore-active peptides, which are of cationic character, and it is thus not discussed in this work. The figure in A is reprinted with permission from PNAS, Volume 105 (Qian et al. 2008b) (C) 2008 National Academy of Sciences, USA

to understand, at molecular level, the requirements for pore induction; for example, they show that a particular type of secondary structure is apparently not strictly needed, since pores are found with disordered, $\alpha$-helical [magainin and melittin (Leontiadou et al. 2006; Sengupta et al. 2008)] and $\beta$-sheet peptides [cateslytin (Jean-François et al. 2008)]. However, the influence of peptide helicity described for the case of magainin (Rzepiela et al. 2009) emphasizes the importance of amphipathicity. In fact, although the peptide charge is determinant, its effect depends on the peptide sequence (Sengupta et al. 2008; Jean-François et al. 2008). Simulations also show that peptide binding prior to pore formation decreases the order of adjacent lipids and increases fluctuations of local thickness (Sengupta et al. 2008). Below, we try to relate the latter perturbations to the capacity of the peptide to reduce the activation energy for the transition to a pore state.

Peptide-driven pore induction: a catalysis-like effect?

As we have discussed, formation of hydrophilic pores in lipid membranes is generally hindered by high activation energies, which manifest as a low frequency of membrane permeability in the absence of perturbation factors. Thus, to interpret pore induction by active peptides, we may consider their role for reducing the energy barrier by way of facilitating lipid reorientation, which is needed to yield the hydrophilic pore (Wohlert et al. 2006; Tieleman and Marrink 2006). The type of local defects from which such lipid reorganization occurs may be regarded as the transition state. Because the peptide binds favorably to the membrane interface, a reduction of the activation energy involves an even more favorable binding affinity of the peptide to the transition state (Fig. 6). This has resemblance to an induced fit (Koshland 1958), and in practice the peptide may be viewed as exerting a catalysis-like transformation of the bilayer from a nonporated to a porated state. We must, however, stress that the concept that we are proposing cannot be assimilated easily into classical catalysis. First, the peptide action also involves stabilization of the pore once it is formed (Fig. 6). This, as we discuss below, is associated with reorganization of the peptide-membrane complex, including a change of pore size, and has the consequence of shifting the equilibrium towards the membrane porated state; i.e., the putative catalyst, the peptide, participates also as a reactant. Secondly, the decrease of the activation energy arguably depends on the amount of membrane-bound peptide.

The nature of the transition state remains undefined. Nevertheless, it may be approximated by a membrane state prone to water defects, similar to those reported in some simulations (Böckmann et al. 2008; Thogersen et al. 2008). The preferential binding of membrane-active peptides for domain boundaries (García-Sáez et al. 2007; Shaw et al. 2008), which are defect-prone regions, would in fact push the membrane towards the transition state. MD simulations also show that binding of membrane-active peptides (magainin and melittin) is accompanied by disorder of neighboring lipids and a nonlinear, $P / L$-dependent increase of thickness fluctuations, which helps to drag the lipid head-groups toward the membrane interior and thus offers a plausible mechanism for reduction of the activation energy (Sengupta et al. 2008).

Formation and evolution of pores: a play in two acts

Huang's model of pore formation assumes a transition between two states on the basis of the changes of peptide 


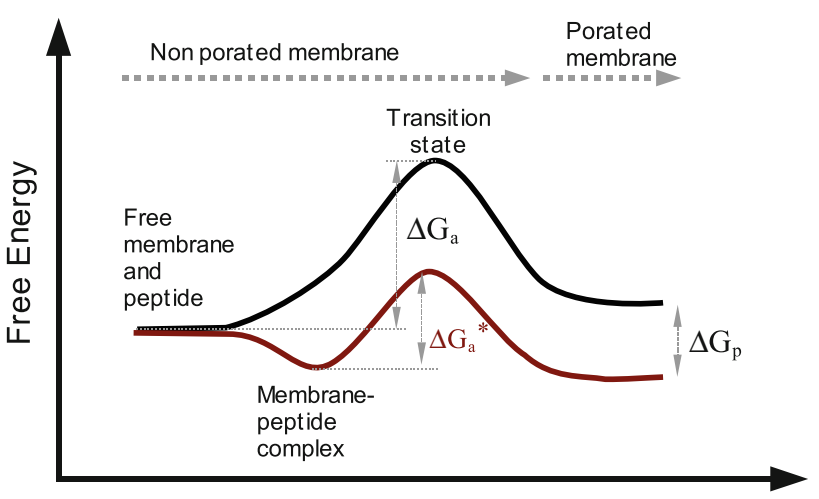

Reaction Coordinate

Fig. 6 Free energy along the reaction coordinate for opening of a lipidic pore coupled to formation and reorganization of a peptide-membrane complex. Starting from peptide and membrane free species an initial membrane-peptide complex is formed favorably, with peptides adsorbed at the interface. As peptides accumulate, the membrane is stretched asymmetrically, causing thinning and increase of fluctuations. Peptides are postulated to bind more strongly in zones of bilayer defects, corresponding to the transition state, which reduces the activation free energy $\left(\Delta G_{\mathrm{a}}\right)$ for lipid reorientation and lipidic pore formation. The $\Delta G_{\mathrm{a}}^{*}$ energy barrier is expected to decrease with increasing the fraction of membrane-bound peptide up to some threshold value. Additionally, after the pore is formed, binding of the peptide near the pore rim stabilizes it via reduction of the line tension. Notice that the first part of the transformation resembles a catalyzed reaction

orientation around a threshold $(P / L)^{*}$ (parallel versus perpendicular to the membrane) and of the membrane thinning reaching a plateau for the same $(P / L)^{*}$ (Huang 2000). This was latter supported for magainin (Tamba and Yamazaki 2005) and melittin (Lee et al. 2008) by kinetic studies using single GUVs. Pore induction by peptides starts with an asymmetric attack to the external (accessible) monolayer (Fig. 7a). The peptide, initially unfolded in water, is driven to fold in the membrane interface due to the more favorable partitioning of hydrogen-bonded peptide groups (Ladokhin and White 1999). As discussed above, the interfacial peptide accumulation causes area stretching and membrane thinning, equivalent to an internal surface tension (Ludtke et al. 1995; Huang et al. 2004). At this early stage, the tension is exerted asymmetrically, just in the accessible monolayer (Gregory et al. 2009; Tamba and Yamazaki 2009). MD simulations suggest (Sengupta et al. 2008; Jean-François et al. 2008) that such an imbalanced perturbation (area expansion and electrical imbalance, arising from the peptide charge) plays a determinant role. The asymmetric tension and lipid fluctuations increase with the amount of bound peptide (Sengupta et al. 2008), and so should also increase the probability for defect formation (Fig. 7b), up to a point where pore formation is no longer kinetically hindered. In an MD study of the stability of membranes under a strongly asymmetric lateral pressure, simulated using a different number of lipids in the two monolayers (Esteban-Martin et al. 2009), it has been shown that, in an asymmetrically expanded bilayer, the packing of the compressed monolayer significantly retards membrane rupture. However, for the case of asymmetric expansion due to binding of peptides in just one monolayer, the situation can be rather different if the peptides exhibit preferential binding to the transition state (i.e., to bilayer defects), since this will lead to a reduction of the activation energy barrier. This is the basis for the proposed catalysislike action introduced above.

Once formed, the pore serves as a mechanism to relieve the initial asymmetric tension (Fig. 7c); but, contrary to what is often assumed, pores corresponding to the initial attack do not close after equilibration. In a careful analysis of successive leakage events in single GUVs porated by Bax- $\alpha 5$, we have recently shown (Fuertes et al. 2010a) that the porated state of vesicles is maintained over long periods of time (Fig. 8) and up to equilibrium conditions. However, initial pores are large, with an estimated radius near $5.8 \mathrm{~nm}$ on the basis of size discrimination for release of dextrans (García-Sáez et al. 2006), and relax to pores of about $2.3 \mathrm{~nm}$ radius (Fuertes et al. 2010a). A reduction of the pore size has been also suggested for melittin (Matsuzaki et al. 1997) and magainin (Tamba et al. 2010). Thus, generalizing to other cationic membrane-active peptides, we may conclude that pores formed immediately after peptide attack are in a transient state that relaxes to smaller pores (Fig. 7d). Pore shrinkage is likely due to the reequilibration of the peptides across the bilayer. Additionally the peptides are able to stabilize the lipidic toroidal pores probably through a reduction of the line tension via interactions at or near the pore edge (Mihajlovic and Lazaridis 2010).

Finally, a few words should be added about the role of peptide aggregation for the induction and stabilization of pores. Although oligomers are not a necessary requirement for the mechanism just described, it is clear that aggregation should facilitate the pore formation process. Aggregates are expected to have stronger capacity to increase membrane fluctuations and drive the appearance of defects, and should thus involve a greater reduction of the activation energy, as suggested by MD simulations (Sengupta et al. 2008; Jean-François et al. 2008). Similarly, proteins should also exhibit a higher specific activity for pore induction than peptides, especially for cases where more than one membrane-active segment exists in the polypeptide chain, and this should be more enhanced even in cases of protein aggregates. For example, the proapoptotic regulator Bax, which acts by porating mitochondria, possesses two potent pore-inducing segments ( $\alpha 5$ and $\alpha 6$ ), both exhibiting independent activity (García-Sáez et al. 2005, 2006; Guillemin et al. 2010; Valero et al. 2011). The individual potency of fragments should add in the full-length 


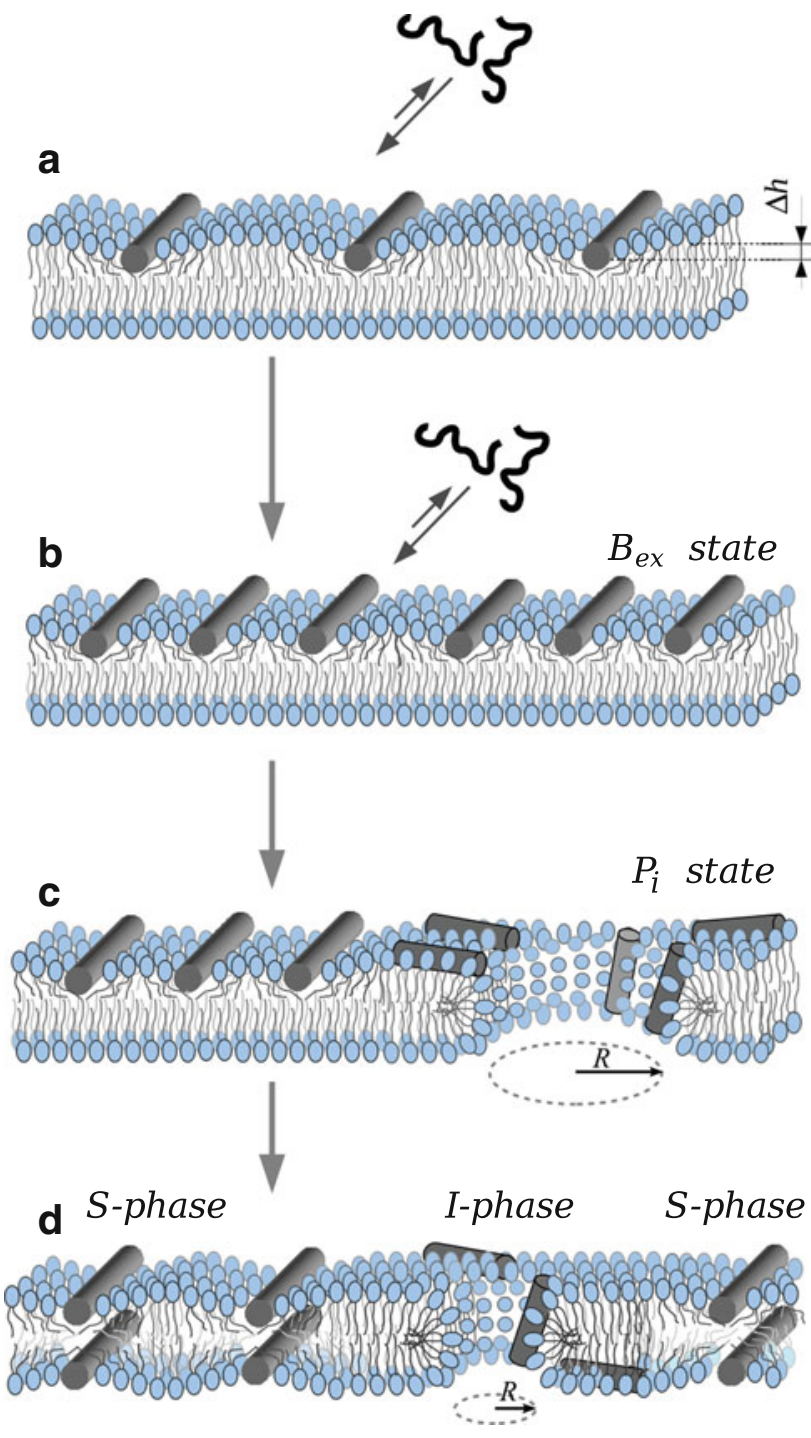

protein, probably with some extra contribution from other membrane-binding parts.

\section{Concluding remarks}

By reviewing defect-driven pore formation in peptide/ protein-free bilayers, both spontaneously and induced by different types of stress, we show here that these pores are clearly connected to pores formed in the presence of membrane-active peptides, up to the point that the latter can be best described as peptide-induced lipid pores. Notwithstanding the importance of a broad family of peptides as true specialists for pore induction, it can be appreciated that the essentials of the pore formation mechanism are shared by the different types of pores. We reason that the long-recognized sealing capacity of biomembranes is mainly due to the background high activation energy for lipidic pore formation, which
4Fig. 7 General mechanism for pore induction by cationic amphipathic peptides. a Pore-active peptides bind avidly to the accessible interface of lipid bilayers, even if they are made of zwitterionic lipids. Peptide binding is coupled to folding (in this case as an $\alpha$-helix, represented by a cylinder). The peptides occupy a volume only in the interfacial region, causing asymmetric area stretching and membrane thinning $(\Delta h)$. As a consequence, fluctuations increase and defects become more likely. b In the so-called $B_{\mathrm{ex}}$ state (Tamba and Yamazaki 2009), membrane perturbation increases with the amount of bound peptides. We postulate (see the text and Fig. 6) that peptides bind with higher affinity near bilayer defects, stabilizing them and so reducing the activation energy for lipid reorganization and pore formation, which would constitute the transition state. c Eventually, the membrane yields and a pore is formed (so-called $P_{\mathrm{i}}$ state by Tamba and Yamazaki). The initial pore is large (Tamba et al. 2010; Fuertes et al. 2010a). As the peptides diffuse through the pore to the opposite monolayer the accumulated asymmetric internal tension diminishes and the pore tends to close due to the line tension at the bilayer edge. However, the peptides bind near the pore rim and reduce the line tension, until an equilibrium is reached with a smaller but stable pore $\mathbf{d}$. The appearance of pores can be understood as a phase transition, and in the equilibrium state two phases coexist (Huang et al. 2004; Huang 2009), namely $S$, with peptides bound essentially parallel to the membrane and lamellar bilayer lipids, and $I$, with peptides exhibiting a certain tilt rear the rim of pores and at the pore wall formed by lipid head groups. The structure of such a toroidal pore when induced by Bax- $\alpha 5$ has been characterized by grazingangle X-ray diffraction (Qian et al. 2008b)

corresponds to a barrier for nucleation of defects. However, defect-prone states such as phase coexistence, and different perturbation factors, including external and internal sources of tension, can lower the barrier and increase pore likelihood. Litster's pore nucleation theory provides the basis for understanding pore stability. Additionally, the mechanism, structure, and dynamics of pores can be described by multiscale MD simulations, which show hydrophobic pre-pore intermediates, first consisting of water files, that grow and convert into a hydrophilic pore lined by lipid head-groups. The same basic framework explains pore induction by peptides. They act as a membrane perturbation factor, similar to other surface-active molecules, by stretching the membrane after interfacial binding. This corresponds to an internal increase of surface tension which decreases the activation energy for pore opening and can thus be considered a type of catalysis. Additionally, the active peptides stabilize the pore by reducing the line tension. The recently determined structure of the hydrophilic toroidal pore induced by the Bax- $\alpha 5$ fragment stands as a demonstration of the peptide-induced lipidic pore hypothesis. This type of pore is a highly dynamic structure. In a biological context it should originate from an external attack to the membrane outer monolayer. Then, it further evolves to an equilibrium state where peptide molecules distributed in the two monolayers stabilize pores of a smaller size. 
a
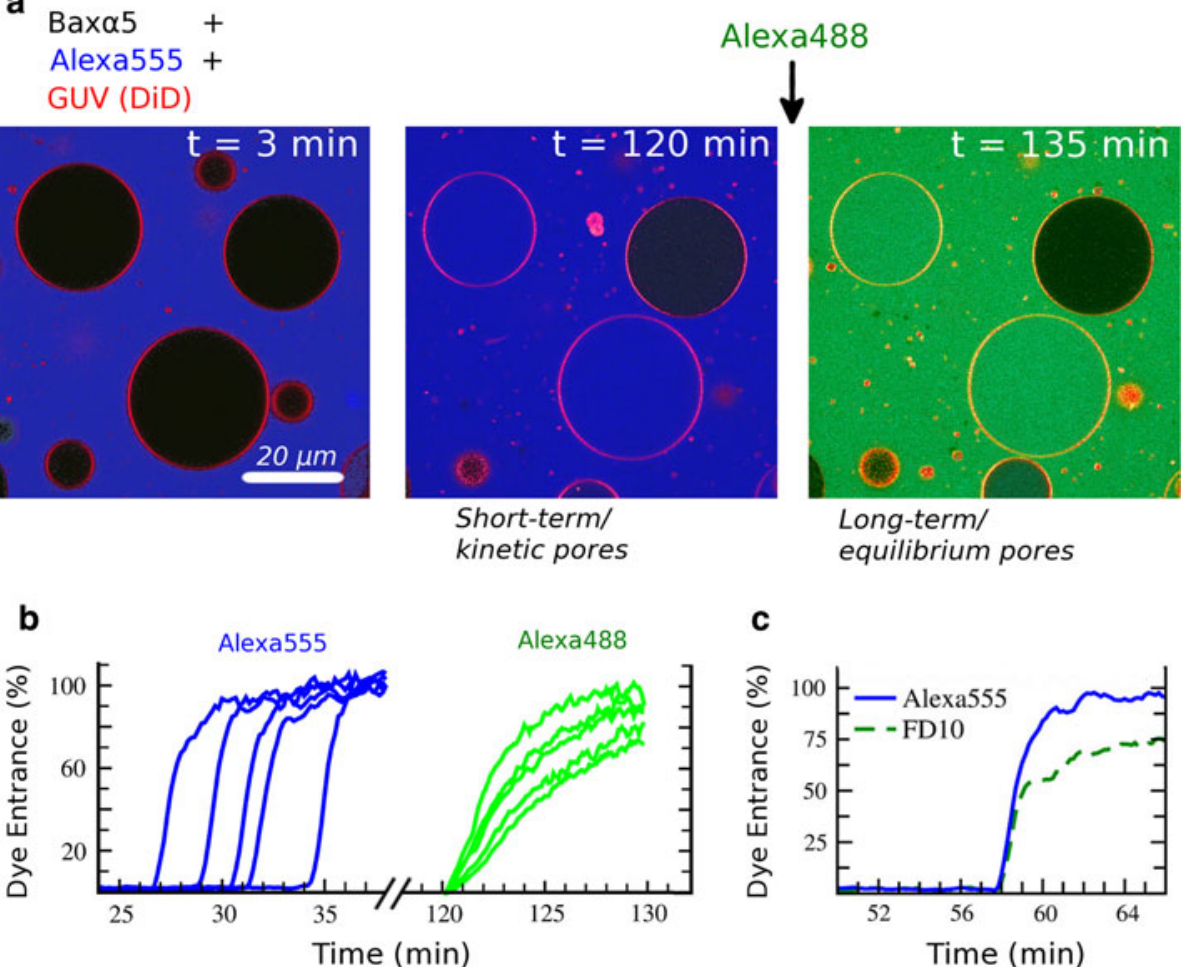

C

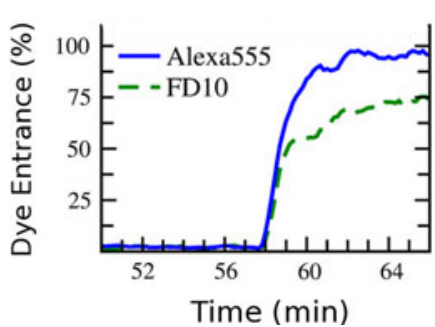

Fig. 8 Shrinkage of pores induced by Bax- $\alpha 5$ on individual GUVs observed by fluorescence microscopy. a Bax- $\alpha 5$ and a first dye (Alexa555, blue) are initially together in the observation chamber, where GUVs (POPC:cardiolipin (CL) 80:20) labeled with 1,1dioctadecyl-3,3,3,3-tetramethylindodicarbocyanine, 4-chlorobenzenesulfonate (DiD) (red) are added. A short time after (left panel), most observed GUVs are not yet porated. The leakage of the blue dye to the inside of GUVs is somewhat retarded and happens stochastically, but once initiated it completes for most GUVs in a few minutes (example kinetics are shown in b, graphs on the left). After $2 \mathrm{~h}$, some GUVs are completely equilibrated with the outside dye while others are empty

Acknowledgments This work was supported by the Spanish MICINN (BFU200767097, financed in part by the European Regional Development Fund) and by Generalitat Valenciana (GVACOMP2010-136). G.F. was a recipient of a grant from the University of Valencia ( $V$ Segles). The Servei d'Informàtica de la Universitat de València is acknowledged for access to the TIRANT supercomputer.

Open Access This article is distributed under the terms of the Creative Commons Attribution Noncommercial License which permits any noncommercial use, distribution, and reproduction in any medium, provided the original author(s) and source are credited.

\section{References}

Abidor I, Arakelyan V, Chernomordik Y, Chizmadzhev V, Tarasevich M (1979) Electrical breakdown ofBLM: main experimental facts and their qualitative discussion. Bioelectrochem Bioenerg $6: 37-52$

Ahmad S, Evans W (2002) Post-translational integration and oligomerization of connexin 26 in plasma membranes and evidence of formation of membrane pores: implications for the assembly of gap junctions. Biochem J 365:693-699 (a, middle panel). If then a second dye is added (Alexa 488, green), internalization occurs immediately and only for the vesicles completely porated at first instance (a, right panel). However, the entrance kinetics for this second leakage event is clearly slower (b, graphs in the right). In an independent experiment $\mathbf{c}$ the entry of Alexa555 (blue) and a fluorescein-labeled dextran of $10 \mathrm{kDa}$ (FD10, green) induced by Bax- $\alpha 5$ on POPC:CL GUVs (80:20) were registered simultaneously by fluorescence microscopy. For any porated GUV, both dyes start leaking in at the same time and at a similar initial rate. However, the entrance of the larger FD10 is drastically slowed down before completion. Data from Fuertes et al. (2010a)

Almeida PF, Pokorny A (2009) Mechanisms of Antimicrobial, Cytolytic, and Cell-Penetrating Peptides: from Kinetics to Thermodynamics. Biochemistry 48:8083-8093. doi:10.1021/bi900914g

Andersen S, Jackson A, Heimburg T (2009) Towards a thermodynamic theory of nerve pulse propagation. Prog Neurobiol 88:104-113. doi:10.1016/j.pneurobio.2009.03.002

Antonov VF, Petrov VV, Molnar AA, Predvoditelev DA, Ivanov AS (1980) The appearance of single-ion channels in unmodified lipid bilayer membranes at the phase transition temperature. Nature 283:585-586

Antonov V, Anosov A, Norik V, Smirnova E (2005) Soft perforation of planar bilayer lipid membranes of dipalmitoylphosphatidylcholine at the temperature of the phase transition from the liquid crystalline to the gel state. Eur Biophys J 34:155-162. doi: 10.1007/s00249-004-0438-8

Avery S, Lloyd D, Harwood J (1995) Temperature-dependent changes in plasma-membrane lipid order and the phagocytotic activity of the amoeba Acanthamoeba castellanii are closely correlated. Biochem J 312:811-816

Benz R, Beckers F, Zimmermann U (1979) Reversible electrical breakdown of lipid bilayer membranes: a charge-pulse relaxation study. J Membr Biol 48:181-204. doi:10.1007/BF01872858

Blicher A, Wodzinska K, Fidorra M, Winterhalter M, Heimburg T (2009) The temperature dependence of lipid membrane 
permeability, its quantized nature, and the influence of anesthetics. Biophys J 96:4581-4591. doi:10.1016/j.bpj.2009.01.062

Bloom M, Evans E, Mouritsen O (1991) Physical-properties of the fluid lipid-bilayer component of cell-membranes-a perspective. Q Rev Biophys 24:293-397

Böckmann RA, de Groot BL, Kakorin S, Neumann E, Grubmüller H (2008) Kinetics, statistics, and energetics of lipid membrane electroporation studied by molecular dynamics simulations. Biophys J 95:1837-1850. doi:10.1529/biophysj.108.129437

Bodles-Brakhop AM, Heller R, Draghia-Akli R (2009) Electroporation for the delivery of DNA-based vaccines and immunotherapeutics: current clinical developments. Mol Ther 17:585-592. doi: $10.1038 / \mathrm{mt} .2009 .5$

Boheim G, Hanke W, Eibl H (1980) Lipid phase transition in planar bilayer membrane and its effect on carrier- and pore-mediated ion transport. Proc Natl Acad Sci USA 77:3403-3407

Chernomordik LV, Melikyan G, Abidor I, Markin V, Chizmadzhev Y (1985) The shape of lipid molecules and monolayer membrane fusion. Biochim Biophys Acta 812:643-655

Contreras F, Basanez G, Alonso A, Herrmann A, Goñi F (2005) Asymmetric addition of ceramides but not dihydroceramides promotes transbilayer (flip-flop) lipid motion in membranes. Biophys J 88:348-359

Daleke D (2007) Phospholipid flippases. J Biol Chem 282:821-825

De Kruijff B, Van Zoelen EJ (1978) Effect of the phase transition on the transbilayer movement of dimyristoyl phosphatidylcholine in unilamellar vesicles. Biochim Biophys Acta 511:105-115

de Kruijff B, van Zoelen EJ, van Deenen LL (1978) Glycophorin facilitates the transbilayer movement of phosphatidylcholine in vesicles. Biochim Biophys Acta 509:537-542

Deamer D, Bramhall J (1986) Permeability of lipid bilayers to water and ionic solutes. Chem Phys Lipids 40:167-188

DeLong E, Yayanos A (1985) Adaptation of the membrane lipids of a deep-sea bacterium to changes in hydrostatic pressure. Science 228:1101-1103

den Otter WK (2009) Free energies of stable and metastable pores in lipid membranes under tension. J Chem Phys 131:205101. doi: $10.1063 / 1.3266839$

Deryagin B, Gutop Y (1962) Theory of the breakdown (rupture) of free films. Kolloidn Zh 24:370-374

Esteban-Martín S, Salgado J (2007) Self-assembling of peptide/ membrane complexes by atomistic molecular dynamics simulations. Biophys J 92:903-912. doi:10.1529/biophysj.106.093013

Esteban-Martín S, Risselada HJ, Salgado J, Marrink SJ (2009) Stability of asymmetric lipid bilayers assessed by molecular dynamics simulations. J Am Chem Soc 131:15194-15202. doi: $10.1021 / \mathrm{ja} 904450 \mathrm{t}$

Evans E, Heinrich V, Ludwig F, Rawicz W (2003) Dynamic tension spectroscopy and strength of biomembranes. Biophys $\mathbf{J}$ 85:2342-2350. doi:10.1016/S0006-3495(03)74658-X

Farago O, Santangelo C (2005) Pore formation in fluctuating membranes. J Chem Phys 122:044901. doi:10.1063/1.1835952

Finkelstein A (1987) Water-Movement Through Lipid Bilayers, Pores, and Plasma-Membranes: Theory and Reality. Wiley Interscience, New York

Freire E, Markello T, Rigell C, Holloway PW (1983) Calorimetric and fluorescence characterization of interactions between cytochrome b5 and phosphatidylcholine bilayers. Biochemistry 22:1675-1680

Fuertes G, García-Sáez AJ, Esteban-Martín S, Giménez D, SánchezMuñoz OL, Schwille P, Salgado J (2010a) Pores formed by Bax $\alpha 5$ relax to a smaller size and keep at equilibrium. Biophys $\mathbf{J}$ 99:2917-2925. doi:10.1016/j.bpj.2010.08.068

Fuertes G, Giménez D, Esteban-Martín S, Garcia-Sáez A, Sánchez O, Salgado J (2010b) Role of membrane lipids for the activity of pore forming peptides and proteins. Adv Exp Med Biol 677:31-55

Gallaher J, Wodzinska K, Heimburg T, Bier M (2010) Ion-channellike behavior in lipid bilayer membranes at the melting transition. Phys Rev E 81. doi: 10.1103/PhysRevE.81.061925

García-Sáez AJ, Coraiola M, Dalla Serra M, Mingarro I, Menestrina G, Salgado J (2005) Peptides derived from apoptotic Bax and Bid reproduce the poration activity of the parent full-length proteins. Biophys J 88:3976-3990. doi:10.1529/biophysj. 104.058008

García-Sáez AJ, Coraiola M, Dalla Serra M, Mingarro I, Müller P, Salgado J (2006) Peptides corresponding to helices 5 and 6 of Bax can independently form large lipid pores. FEBS J 273:971-981. doi:EJB5123

García-Sáez AJ, Chiantia S, Salgado J, Schwille P (2007) Pore formation by a Bax-derived peptide: effect on the line tension of the membrane probed by AFM. Biophys J 93:103-112. doi: PMC1914428

García-Sáez AJ, Ries J, Orzáez M, Pérez-Payà E, Schwille P (2009) Membrane promotes tBID interaction with BCL(XL). Nat Struct Mol Biol. doi: 10.1038/nsmb.1671

Glaser RW, Leikin SL, Chernomordik LV, Pastushenko VF, Sokirko AI (1988) Reversible electrical breakdown of lipid bilayers: formation and evolution of pores. Biochim Biophys Acta 940:275-287

Gregory SM, Cavenaugh A, Journigan V, Pokorny A, Almeida PFF (2008) A quantitative model for the all-or-none permeabilization of phospholipid vesicles by the antimicrobial peptide cecropin A. Biophys J 94:1667-1680. doi:10.1529/biophysj.107.118760

Gregory SM, Pokorny A, Almeida PFF (2009) Magainin 2 revisited: $S$ test of the quantitative model for the all-or-none permeabilization of phospholipid vesicles. Biophys J 96:116-131. doi: 10.1016/j.bpj.2008.09.017

Guillemin Y, López J, Giménez D, Fuertes G, García Valero J, Blum L, Gonzalo P, Salgado J, Girard-Egrot A, Aouacheria A (2010) Synthetic peptides derived from pro- and anti-apoptotic BCL-2 family members have distinct membrane behaviour reflecting functional divergence. PLoS ONE 5:e9066. doi:10.1371/journal. pone.0009066

Gurtovenko AA, Anwar J (2007) Modulating the Structure and Properties of Cell Membranes: the Molecular Mechanism of Action of Dimethyl Sulfoxide. J Phys Chem B 111:1045310460. doi:10.1021/jp073113e

Gurtovenko AA, Vattulainen I (2005) Pore formation coupled to ion transport through lipid membranes as induced by transmembrane ionic charge imbalance: atomistic molecular dynamics study. J Am Chem Soc 127:17570-17571. doi:10.1021/ja053129n

Gurtovenko A, Vattulainen I (2007a) Molecular mechanism for lipid flip-flops. J Phys Chem B 111:13554-13559

Gurtovenko A, Vattulainen I (2007b) Lipid transmembrane asymmetry and intrinsic membrane potential: two sides of the same coin. J Am Chem Soc 129:5358-5359

Gurtovenko AA, Vattulainen I (2007c) Ion leakage through transient water pores in protein-free lipid membranes driven by transmembrane ionic charge imbalance. Biophys J 92:1878-1890. doi:10.1529/biophysj.106.094797

Gurtovenko AA, Anwar J, Vattulainen I (2010) Defect-Mediated Trafficking across Cell Membranes: insights from in Silico Modeling. Chem Rev 110:6077-6103. doi:10.1021/cr1000783

Heimburg T (2007) Thermal biophysics of membranes. Wiley-VCH, Berlin

Heimburg T (2010) Lipid ion channels. Biophys Chem 150:2-22. doi: 10.1016/j.bpc.2010.02.018

Heimburg T, Jackson A (2005) On soliton propagation in biomembranes and nerves. Proc Natl Acad Sci USA 102:9790-9795 
Heimburg T, Jackson A (2007) On the action potential as a propagating density pulse and the role of anesthetics. Biophys Rev Lett 2:57-78

Huang HW (2000) Action of Antimicrobial Peptides: two-State Model. Biochemistry 39:8347-8352. doi:10.1021/bi0009461

Huang HW (2009) Free energies of molecular bound states in lipid bilayers: lethal concentrations of antimicrobial peptides. Biophys J 96:3263-3272. doi:10.1016/j.bpj.2009.01.030

Huang HW, Chen F, Lee M (2004) Molecular mechanism of Peptideinduced pores in membranes. Phys Rev Lett 92:198304

Ivanova V, Makarov I, Schäffer T, Heimburg T (2003) Analyzing heat capacity profiles of peptide-containing membranes: cluster formation of Gramicidin A. Biophys J 84:2427-2439. doi: 10.1016/S0006-3495(03)75047-4

Jansen M, Blume A (1995) A comparative study of diffusive and osmotic water permeation across bilayers composed of phospholipids with different head groups and fatty acyl chains. Biophys J 68:997-1008

Jean-François F, Elezgaray J, Berson P, Vacher P, Dufourc EJ (2008) Pore formation induced by an antimicrobial peptide: electrostatic effects. Biophys J 95:5748-5756. doi:10.1529/biophysj.108. 136655

Jensen MØ, Mouritsen OG (2004) Lipids do influence protein function-the hydrophobic matching hypothesis revisited. Biochim Biophys Acta 1666:205-226. doi:10.1016/j.bbamem.2004. 06.009

Kandasamy SK, Larson RG (2006) Cation and anion transport through hydrophilic pores in lipid bilayers. J Chem Phys 125:074901. doi:10.1063/1.2217737

Kaufmann K, Hanke W, Corcia A (1989) Ion channel fluctuations in pure lipid bilayer membranes: control by voltage. Caruaru

Kol MA, de Kroon AI, Rijkers DT, Killian JA, de Kruijff B (2001) Membrane-spanning peptides induce phospholipid flop: a model for phospholipid translocation across the inner membrane of $\mathrm{E}$. coli. Biochemistry 40:10500-10506

Kol MA, van Dalen A, de Kroon AIPM, de Kruijff B (2003) Translocation of phospholipids is facilitated by a subset of membrane-spanning proteins of the bacterial cytoplasmic membrane. J Biol Chem 278:24586-24593. doi:10.1074/jbc.M3018 75200

Kornberg RD, McConnell HM (1971) Inside-outside transitions of phospholipids in vesicle membranes. Biochemistry 10:1111-1120

Koshland DJ (1958) Application of a theory of enzyme specificity to protein synthesis. Proc Natl Acad Sci USA 44:105-112

Ladokhin AS, White SH (1999) Folding of amphipathic alpha-helices on membranes: energetics of helix formation by melittin. J Mol Biol 285:1363-1369. doi:10.1006/jmbi.1998.2346

Lee A (2004) How lipids affect the activities of integral membrane proteins. Biochim Biophys Acta 1666:62-87

Lee M, Hung W, Chen F, Huang H (2008) Mechanism and kinetics of pore formation in membranes by water-soluble amphipathic peptides. Proc Natl Acad Sci USA 105:5087-5092. doi: 10.1073/pnas.0710625105

Leontiadou H, Mark AE, Marrink SJ (2004) Molecular dynamics simulations of hydrophilic pores in lipid bilayers. Biophys $\mathrm{J}$ 86:2156-2164

Leontiadou H, Mark AE, Marrink SJ (2006) Antimicrobial peptides in action. J Am Chem Soc 128:12156-12161. doi:10.1021/ja062927q

Litster JD (1975) Stability of lipid bilayers and red blood cell membranes. Phys Lett A 53:193-194. doi:10.1016/0375-9601(75) 90402-8

Longo M, Waring A, Gordon L, Hammer D (1998) Area expansion and permeation of phospholipid membrane bilayers by influenza fusion peptides and melittin. Langmuir 14:2385-2395

Ludtke S, He K, Huang H (1995) Membrane thinning caused by magainin 2. Biochemistry 34:16764-16769
Ludtke SJ, He K, Heller WT, Harroun TA, Yang L, Huang HW (1996) Membrane pores induced by magainin. Biochemistry 35:13723-13728. doi:10.1021/bi9620621

Marrink SJ, Lindahl E, Edholm O, Mark AE (2001) Simulation of the spontaneous aggregation of phospholipids into bilayers. J Am Chem Soc 123:8638-8639

Marrink SJ, de Vries AH, Tieleman DP (2009) Lipids on the move: simulations of membrane pores, domains, stalks and curves. Biochim Biophys Acta 1788:149-168. doi:10.1016/j.bbamem. 2008.10.006

Mathai J, Missner A, Gler P, Saparov S, Zeidel M, Lee J, Pohl P (2009) No facilitator required for membrane transport of hydrogen sulfide. Proc Natl Acad Sci USA 106:16633-16638

Matsuzaki K, Murase O, Fujii N, Miyajima K (1996) An antimicrobial peptide, magainin 2, induced rapid flip-flop of phospholipids coupled with pore formation and peptide translocation. Biochemistry 35:11361-11368. doi:10.1021/bi960016v

Matsuzaki K, Yoneyama S, Miyajima K (1997) Pore formation and translocation of melittin. Biophys J 73:831-838. doi:10.1016/ S0006-3495(97)78115-3

Meierhenrich U, Filippi J, Meinert C, Vierling P, Dworkin J (2010) On the origin of primitive cells: from nutrient intake to elongation of encapsulated nucleotides. Angew Chem Int Ed Engl 49:3738-3750

Melikov KC, Frolov VA, Shcherbakov A, Samsonov AV, Chizmadzhev YA, Chernomordik LV (2001) Voltage-induced nonconductive pre-pores and metastable single pores in unmodified planar lipid bilayer. Biophys J 80:1829-1836

Mihajlovic M, Lazaridis T (2010) Antimicrobial peptides bind more strongly to membrane pores. Biochim Biophys Acta 1798:1494 1502. doi:10.1016/j.bbamem.2010.02.023

Mills J, Needham D (2005) Lysolipid incorporation in dipalmitoylphosphatidylcholine bilayer membranes enhances the ion permeability and drug release rates at the membrane phase transition. Biochim Biophys Acta 1716:77-96. doi:10.1016/j.bbamem.2005.08.007

Moldovan D, Pinisetty D, Devireddy RV (2007) Molecular dynamics simulation of pore growth in lipid bilayer membranes in the presence of edge-active agents. Appl Phys Lett 91:204104. doi: $10.1063 / 1.2814876$

Moroz JD, Nelson P (1997) Dynamically stabilized pores in bilayer membranes. Biophys J 72:2211-2216. doi:10.1016/S00063495(97)78864-7

Morrow MR, Davis JH, Sharom FJ, Lamb MP (1986) Studies on the interaction of human erythrocyte band 3 with membrane lipids using deuterium nuclear magnetic resonance and differential scanning calorimetry. Biochim Biophys Acta 858:13-20

Nagle J, Scott H Jr (1978) Lateral compressibility of lipid mono- and bilayers. Theory of membrane permeability. Biochim Biophys Acta 513:236-243

Nagle J, Mathai J, Zeidel M, Tristram-Nagle S (2008) Theory of passive permeability through lipid bilayers. J Gen Physiol 131:77-85

Nakano M, Fukuda M, Kudo T, Matsuzaki N, Azuma T, Sekine K, Endo H, Handa T (2009) Flip-flop of phospholipids in vesicles: kinetic analysis with time-resolved small-angle neutron scattering. J Phys Chem B 113:6745-6748. doi:10.1021/jp900913w

Neumann E, Schaefer-Ridder M, Wang Y, Hofschneider PH (1982) Gene transfer into mouse lyoma cells by electroporation in high electric fields. EMBO J 1:841-845

Papahadjopoulos K, Jacobson K, Nir S, Isac T (1973) Phasetransitions in phospholipid vesicles - fluorescence polarization and permeability measurements concerning effect of temperature and cholesterol. Biochim Biophys Acta 311:330-348. doi: 10.1016/0005-2736(73)90314-3

Paula S, Volkov A, Van Hoek A, Haines T, Deamer D (1996) Permeation of protons, potassium ions, and small polar 
molecules through phospholipid bilayers as a function of membrane thickness. Biophys J 70:339-348

Phillips R, Ursell T, Wiggins P, Sens P (2009) Emerging roles for lipids in shaping membrane-protein function. Nature 459:379-385

Puech P, Borghi N, Karatekin E, Brochard-Wyart F (2003) Line Thermodynamics: adsorption at a Membrane Edge. Phys Rev Lett 90:128304. doi:10.1103/PhysRevLett.90.128304

Qian S, Wang W, Yang L, Huang HW (2008a) Structure of the alamethicin pore reconstructed by x-ray diffraction analysis. Biophys J 94:3512-3522. doi:10.1529/biophysj.107.126474

Qian S, Wang W, Yang L, Huang HW (2008b) Structure of transmembrane pore induced by Bax-derived peptide: evidence for lipidic pores. Proc Natl Acad Sci USA 105:17379-17383. doi:10.1073/pnas.0807764105

Rathinakumar R, Wimley WC (2008) Biomolecular engineering by combinatorial design and high-throughput screening: small, soluble peptides that permeabilize membranes. J Am Chem Soc 130:9849-9858. doi:10.1021/ja8017863

Ruiz-Argüello M, Basáñez G, Goñi F, Alonso A (1996) Different effects of enzyme-generated ceramides and diacylglycerols in phospholipid membrane fusion and leakage. J Biol Chem 271:26616-26621

Ryttsén F, Farre C, Brennan C, Weber SG, Nolkrantz K, Jardemark K, Chiu DT, Orwar O (2000) Characterization of single-cell electroporation by using patch-clamp and fluorescence microscopy. Biophys J 79:1993-2001

Rzepiela A, Sengupta D, Goga N, Marrink S (2009) Membrane poration by antimicrobial peptides combining atomistic and coarse-grained descriptions. Faraday Discuss 144:431-443

Sandre O, Moreaux L, Brochard-Wyart F (1999) Dynamics of transient pores in stretched vesicles. Proc Natl Acad Sci USA 96:10591-10596

Sapay N, Bennett W, Tieleman D (2010) Molecular simulations of lipid flip-flop in the presence of model transmembrane helices. Biochemistry 49:7665-7673

Schwarz S, Haest CW, Deuticke B (1999) Extensive electroporation abolishes experimentally induced shape transformations of erythrocytes: a consequence of phospholipid symmetrization? Biochim Biophys Acta 1421:361-379

Seeger HM, Fidorra M, Heimburg T (2005) Domain size and fluctuations at domain interfaces in lipid mixtures. Macromol Symp 219:85-96. doi:10.1002/masy.200550109

Sengupta D, Leontiadou H, Mark AE, Marrink S (2008) Toroidal pores formed by antimicrobial peptides show significant disorder. Biochim Biophys Acta 1778:2308-2317. doi:S0005-2736(08) 00176-4

Shaw J, Epand R, Hsu J, Mo G, Epand R, Yip C (2008) Cationic peptide-induced remodelling of model membranes: direct visualization by in situ atomic force microscopy. J Struct Biol 162:121-138. doi:10.1016/j.jsb.2007.11.003

Shillcock JC, Boal DH (1996) Entropy-driven instability and rupture of fluid membranes. Biophys J 71:317-326. doi:10.1016/ S0006-3495(96)79227-5

Simons K, Vaz WLC (2004) Model systems, lipid rafts, and cell membranes. Annu Rev Biophys Biomol Struct 33:269-295. doi: 10.1146/annurev.biophys.32.110601.141803

Singer SJ, Nicolson GL (1972) The fluid mosaic model of the structure of cell membranes. Science 175:720-731

Siskind LJ, Colombini M (2000) The lipids C2- and C16-ceramide form large stable channels. Implications for apoptosis. J Biol Chem 275:38640-38644. doi:10.1074/jbc.C000587200

Tamba Y, Yamazaki M (2005) Single giant unilamellar vesicle method reveals effect of antimicrobial peptide magainin 2 on membrane permeability. Biochemistry 44:15823-15833. doi: 10.1021/bi051684w
Tamba Y, Yamazaki M (2009) Magainin 2-induced pore formation in the lipid membranes depends on its concentration in the membrane interface. J Phys Chem B 113:4846-4852. doi: 10.1021/jp8109622

Tamba Y, Ariyama H, Levadny V, Yamazaki M (2010) Kinetic pathway of antimicrobial peptide magainin 2-induced pore formation in lipid membranes. J Phys Chem B 114:1201812026. doi:10.1021/jp104527y

Tarek M (2005) Membrane electroporation: a molecular dynamics simulation. Biophys J 88:4045-4053. doi:10.1529/biophysj.104. 050617

Taupin C, Dvolaitzky M, Sauterey C (1975) Osmotic pressure induced pores in phospholipid vesicles. Biochemistry 14:4771-4775

Thogersen L, Schiott B, Vosegaard T, Nielsen N, Tajkhorshid E (2008) Peptide aggregation and pore formation in a lipid bilayer: a combined coarse-grained and all atom molecular dynamics study. Biophys J 95:4337-4347. doi:10.1529/biophysj.108.133330

Tieleman DP, Marrink S (2006) Lipids out of equilibrium: energetics of desorption and pore mediated flip-flop. J Am Chem Soc 128:12462-12467. doi:10.1021/ja0624321

Tieleman DP, Leontiadou H, Mark AE, Marrink S (2003) Simulation of pore formation in lipid bilayers by mechanical stress and electric fields. J Am Chem Soc 125:6382-6383. doi:10.1021/ja029504i

Tolpekina TV, den Otter WK, Briels WJ (2004a) Nucleation free energy of pore formation in an amphiphilic bilayer studied by molecular dynamics simulations. J Chem Phys 121:1206012066. doi:10.1063/1.1815296

Tolpekina TV, den Otter WK, Briels WJ (2004b) Simulations of stable pores in membranes: system size dependence and line tension. J Chem Phys 121:8014. doi:10.1063/1.1796254

Toyoshima Y, Thompson TE (1975) Chloride flux in bilayer membranes: chloride permeability in aqueous dispersions of single-walled, bilayer vesicles. Biochemistry 14:1525-1531

Tsong TY (1991) Electroporation of cell membranes. Biophys J 60:297-306. doi:10.1016/S0006-3495(91)82054-9

Valero JG, Sancey L, Kucharczak J, Guillemin Y, Gimenez D, Prudent J, Gillet G, Salgado J, Coll J, Aouacheria A (2011) Baxderived membrane-active peptides act as potent and direct inducers of apoptosis in cancer cells. J Cell Sci 124:556-564. doi: $10.1242 /$ jcs.076745

Weinstein J, Klausner R, Innerarity T, Ralston E, Blumenthal R (1981) Phase-transition release, a new approach to the interaction of proteins with lipid vesicles. Application to lipoproteins. Biochim Biophys Acta 647:270-284

White SH, Wimley WC (1999) Membrane protein folding and stability: physical principles. Annu Rev Biophys Biomol Struct 28:319-365

White SH, Ladokhin AS, Jayasinghe S, Hristova K (2001) How membranes shape protein structure. J Biol Chem 276:32395-32398. doi:10.1074/jbc.R100008200

Wiener MC, White SH (1991) Fluid bilayer structure determination by the combined use of $\mathrm{x}$-ray and neutron diffraction. I. Fluid bilayer models and the limits of resolution. Biophys J 59:162-173

Wiener MC, White SH (1992) Structure of a fluid dioleoylphosphatidylcholine bilayer determined by joint refinement of $\mathrm{x}$-ray and neutron diffraction data III. Complete structure. Biophys J 61:434-447. doi:10.1016/S0006-3495(92)81849-0

Wilson M, Pohorille A (1996) Mechanism of unassisted ion transport across membrane bilayers. J Am Chem Soc 118:6580-6587

Wimley WC (2010) Describing the mechanism of antimicrobial peptide action with the interfacial activity model. ACS Chem Biol 5:905-917. doi:10.1021/cb1001558

Wimley WC, Thompson TE (1990) Exchange and flip-flop of dimyristoylphosphatidylcholine in liquid-crystalline, gel, and two-component, two-phase large unilamellar vesicles. Biochemistry $29: 1296-1303$ 
Wimley WC, Thompson TE (1991) Transbilayer and interbilayer phospholipid exchange in dimyristoylphosphatidylcholine/ dimyristoylphosphatidylethanolamine large unilamellar vesicles. Biochemistry 30:1702-1709. Retrieved 4 May, 2009, from http://www.ncbi.nlm.nih.gov/pubmed/1993185

Wodzinska K, Blicher A, Heimburg T (2009) The thermodynamics of lipid ion channel formation in the absence and presence of anesthetics. BLM experiments and simulations. Soft Matter 5:3319-3330. doi:10.1039/b909877a

Wohlert J, den Otter W, Edholm O, Briels W (2006) Free energy of a trans-membrane pore calculated from atomistic molecular dynamics simulations. J Chem Phys 124. doi: 10.1063/1.2171965

Yafuso M, Kennedy S, Freeman A (1974) Spontaneous conductance changes, multilevel conductance states and negative differential resistance in oxidized cholesterol black lipid membranes. J Membr Biol 17:201-212
Yandek LE, Pokorny A, Florén A, Knoelke K, Langel U, Almeida PFF (2007) Mechanism of the cell-penetrating peptide transportan 10 permeation of lipid bilayers. Biophys J 92:2434-2444. doi:10.1529/biophysj.106.100198

Yoshikawa K, Fujimoto T, Shimooka T, Terada H, Kumazawa N, Ishii T (1988) Electrical oscillation and fluctuation in phospholipid-membranes. Phospholipids can form a channel without protein. Biophys Chem 29:293-299

Zasloff M (2002) Antimicrobial peptides of multicellular organisms. Nature 415:389-395. doi:10.1038/415389a

Zhelev DV, Needham D (1993) Tension-stabilized pores in giant vesicles: determination of pore size and pore line tension. Biochim Biophys Acta 1147:89-104 